\title{
Optimal Weight Gain During Pregnancy in Japanese Women
}

\author{
Shunji Suzuki
}

\begin{abstract}
Background: In order to examine the optimal weight gain during pregnancy in Japanese women, we analyzed the perinatal outcomes in Japanese women with the optimal range of weight gain during pregnancy according to the Japanese (the Japanese Ministry of Health, Labour and Welfare: JMHLW and the Japan Society for the Study of Obesity: JASSO) guidelines compared with those according to the USA (the Institute of Medicine: IOM) guideline.
\end{abstract}

Methods: We compared the obstetric outcomes in two groups of gestational weight gain within the optimal range based on the IOM and Japanese guidelines in women of pre-pregnancy body mass index (BMI) categories of underweight, normal, overweight and obese.

Results: In the underweight and normal-weight women, the incidences of preterm delivery and low-birth-weight infant in the JMHLW group were significantly higher than those in the IOM group; however, the incidence of some other perinatal complications in the JMHLW group was significantly lower than that in the IOM group. In the overweight women, the incidences of preterm delivery and low-birth-weight infant in the JSSO group were significantly higher than those in the IOM group; however, there were no significant differences in the obstetric outcomes between the obese women in the JSSO and IOM groups.

Conclusion: Based on the current results, we should be more tolerant for the weight gain during pregnancy in Japanese woman than ever, especially in overweight women.

Keywords: Optimal weight gain; Body mass index; Japanese women; Pregnancy; Guideline

\section{Introduction}

In the Institute of Medicine (IOM) guideline in 2009, optimal

Manuscript accepted for publication September 02, 2016

Department of Obstetrics and Gynecology, Japanese Red Cross Katsushika Maternity Hospital, 5-11-12 Tateishi, Katsushika-ku, Tokyo 124-0012, Japan. Email:czg83542@mopera.ne.jp

doi: http://dx.doi.org/10.14740/jocmr2723w range of weight gain during pregnancy has been recommended based on the body mass index (BMI; $\mathrm{kg} / \mathrm{m}^{2}$ ) of women during pre-pregnancy such as underweight $(\mathrm{BMI}<18.5)$, normal (BMI: 18.5 - 24.9), overweight (BMI: 25.0 - 29.9), and obese $(\mathrm{BMI} \geq 30)$ [1]. In a recent study in Japan by Enomoto et al [2], the BMI classification by the IOM guideline seemed to be valid in pregnant Japanese women associated with the different incidences of adverse pregnancy outcomes. In their observation, optimal weight gain recommended by the IOM guideline also seemed to be associated with a better outcome in all pre-pregnancy BMI category groups. For example, excess gestational weight gain was associated with a higher frequency of large for gestational age and macrosomia, while poor weight gain was correlated with a higher frequency of small for gestational age [2].

To date, the recommended weight gain during pregnancy for Japanese women has been smaller than that in the IOM guideline in 2009 [3-5], although the BMI classification during pre-pregnancy seemed to be the same between the IOM and Japanese guidelines [1, 3-5]. In the IOM guideline, optimal ranges of weight gain during pregnancy in underweight, normal, overweight and obese women have been reported to be $12.7-18.1,11.3-15.9,6.8-11.3$ and $5.0-9.1 \mathrm{~kg}$, respectively [1]. However, in the Japanese Ministry of Health, Labour and Welfare (JMHLW) guideline, optimal ranges of weight gain during pregnancy in underweight and normal women have been recommended to be $9-12$ and $7-12 \mathrm{~kg}$, respectively [4]. In addition, in the Japan Society for the Study of Obesity (JAS$\mathrm{SO}$ ) guideline, optimal ranges of weight gain during pregnancy in overweight and obese women have been recommended to be $\leq 7$ and $\leq 5 \mathrm{~kg}$, respectively [5]. The average physique of Japanese women is certainly short and slender in comparison to that of the Western woman; however, the studies about the recommended value of weight gain during pregnancy in compliance with the Japanese women have not been well documented $[1,6]$. Therefore, in the current study, we examined the perinatal outcomes in Japanese women with the optimal range of weight gain during pregnancy in the JMHLW and JASSO guidelines compared with those in the IOM guideline.

\section{Methods}

The protocol for this study was approved by the Ethics Committee of the Japanese Red Cross Katsushika Maternity Hospital. Informed consent concerning analysis from a retrospective database was obtained from all subjects. 
Table 1. Clinical Characteristics of the Underweight Women With the Optimal Weight Gain Recommended in the JMHLW and IOM Guidelines

\begin{tabular}{|c|c|c|}
\hline \multirow{2}{*}{ Characteristics } & \multicolumn{2}{|c|}{ Weight gain during pregnancy } \\
\hline & 9 - 12 kg (JMHLW guideline) & $12.7-18.1 \mathrm{~kg}$ (IOM guideline) \\
\hline Number & 204 & 127 \\
\hline \multicolumn{3}{|l|}{ Maternal age } \\
\hline$<20$ years old & $1(0.5 \%)^{*}$ & $5(3.9 \%)$ \\
\hline$\geq 40$ years old & $27(13.2 \%)^{*}$ & $7(5.5 \%)$ \\
\hline Primiparous women & $104(51.0 \%)^{*}$ & $80(63.0 \%)$ \\
\hline Maternal height $(\mathrm{cm})$ & $159 \pm 6.3$ & $159 \pm 4.9$ \\
\hline Maternal weight at pre-pregnancy (kg) & $43.7 \pm 3.8$ & $43.4 \pm 3.3$ \\
\hline Body mass index at pre-pregnancy & $17.2 \pm 0.7$ & $17.2 \pm 0.8$ \\
\hline Maternal weight at delivery $(\mathrm{kg})$ & $54.2 \pm 4.2^{*}$ & $58.1 \pm 3.8$ \\
\hline
\end{tabular}

Data are presented as mean \pm standard deviation or number (percentages). ${ }^{*} \mathrm{P}<0.05$ vs. IOM group. JMHLW: Japanese Ministry of Health, Labour and Welfare; IOM: Institute of Medicine.

We reviewed the obstetric records of singleton pregnant Japanese women who delivered at our institute at $\geq 22$ weeks' gestation from April 2012 through March 2016.

In order to examine the optimal weight gain during pregnancy in Japanese women, we compared the obstetric outcomes in two groups of gestational weight gain within the optimal range based on the IOM and JMHLW guidelines in women of pre-pregnancy BMI categories of underweight and normal weight, and in two groups of gestational weight gain within the optimal range based on the IOM and JASSO guidelines in women of pre-pregnancy BMI categories of overweight and obese.

As characteristics of patients, we examined maternal age, primiparous rate, height, body weight and BMI during prepregnancy, body weight at delivery and total weight gain during pregnancy. The main obstetric outcomes were hypertensive disorders, gestational diabetes mellitus (GDM), macrosomia, low birth weight, preterm delivery, cesarean delivery and postpartum hemorrhage $\geq 1,000 \mathrm{~mL}$. GDM was diagnosed when at least one of the following was found: fasting blood glucose level of $\geq 92 \mathrm{mg} / \mathrm{dL}$, blood glucose level at $1 \mathrm{~h}$ of $\geq 180 \mathrm{mg} / \mathrm{dL}$, and blood glucose level at $2 \mathrm{~h} \geq 153 \mathrm{mg} / \mathrm{dL}$ by 75 -g oral glucose tolerance test. Macrosomia was defined as a neonatal birth weight of $\geq 4,000 \mathrm{~g}$, while low birth weight was defined as a neonatal birth weight of $<2,500 \mathrm{~g}$. Gestational age was calculated based on the ultrasonographic findings at 9 - 11 weeks' gestation.

Data were expressed as mean \pm standard deviation (SD) or number (percentages). Cases and controls were compared by means of Student's $t$-test for continuous variables, and the $\chi^{2}$ or Fisher's exact test for categorical variables. Odds ratios (ORs) and $95 \%$ confidence intervals (CIs) were also calculated. Differences with $\mathrm{P}<0.05$ were considered significant.

\section{Results}

There were 5,351 women who delivered singleton neonates at $\geq 22$ weeks' gestation at our institute during the study pe- riod. Of these, $515(9.6 \%), 4,158(77.7 \%), 555(10.4 \%)$ and $123(2.3 \%)$ women were defined as underweight, normal weight, overweight and obese based on the BMI during prepregnancy, respectively. Of the 515 underweight women, the weight gain during pregnancy was $9-12 \mathrm{~kg}$ (recommended weight gain in the JMHLW guideline) in 204 women (39.6\%), while it was $12.7-18.1 \mathrm{~kg}$ (recommended weight gain in the IOM guideline) in 127 women (24.7\%). Of the 4,158 normalweight women, the weight gain during pregnancy was $7-12$ $\mathrm{kg}$ (recommended weight gain in the JMHLW guideline) in 2,136 women $(51.4 \%$ ), while it was 11.3 - $15.9 \mathrm{~kg}$ (recommended weight gain in the IOM guideline) in 1,348 women $(32.4 \%)$. Of the 565 overweight women, the weight gain during pregnancy was $<7 \mathrm{~kg}$ (recommended weight gain in the JSSO guideline) in 141 women (25.4\%), while it was $6.8-11.3$ $\mathrm{kg}$ (recommended weight gain in the IOM guideline) in 161 women $(29.0 \%)$. Of the 123 obese women, the weight gain during pregnancy was $<5 \mathrm{~kg}$ (recommended weight gain in the JSSO guideline) in 61 women (49.6\%), while it was 5.0 $9.1 \mathrm{~kg}$ (recommended weight gain in the IOM guideline) in 41 women $(33.3 \%)$.

Table 1 shows the characteristics of the underweight women with the optimal weight gain recommended in the JMHLW and IOM guidelines (JMHLW and IOM groups). The ages of the women in the JMHLW group were lower than those in the IOM group. Table 2 shows the perinatal outcomes of the underweight women with the optimal weight gain recommended in the JMHLW and IOM guidelines (JMHLW and IOM groups). The incidence of GDM in the JMHLW group was significantly lower than that in the IOM group, while the incidences of preterm delivery and low-birth-weight infant in the JMHLW group were significantly higher than those in the IOM group.

Table 3 shows the characteristics of the normal-weight women with the optimal weight gain recommended in the JMHLW and IOM guidelines (JMHLW and IOM groups). The rate of women of $\geq 40$ years old in the JMHLW group was lower than that in the IOM group. Table 4 shows the perinatal outcomes of the normal-weight women with the optimal 
Table 2. Perinatal Outcomes of the Underweight Women With the Optimal Weight Gain Recommended in the JMHLW and IOM Guidelines

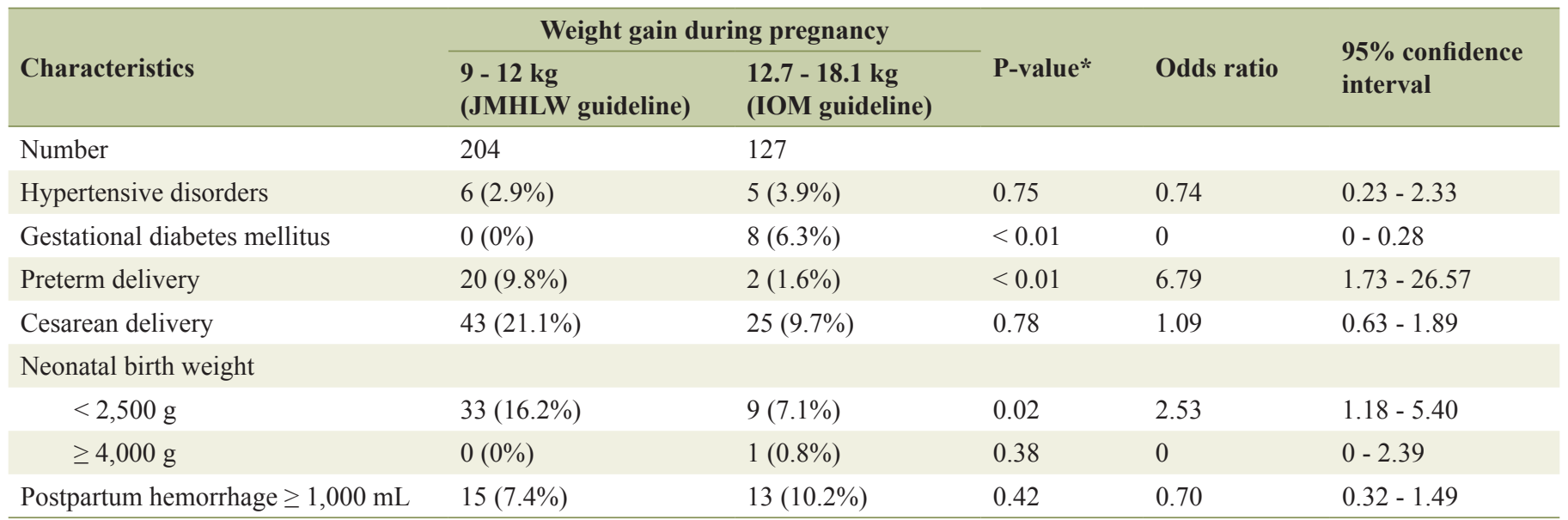

Data are presented as number (percentages). ${ }^{*} \mathrm{P}<0.05$ vs. IOM group. JMHLW: Japanese Ministry of Health, Labour and Welfare; IOM: Institute of Medicine.

weight gain recommended in the JMHLW and IOM guidelines (JMHLW and IOM groups). The incidences of hypertensive disorders, macrosomia and postpartum hemorrhage $\geq 1,000$ $\mathrm{mL}$ in the JMHLW group were significantly lower than those in the IOM group, while the incidences of preterm delivery and low-birth-weight infant in the JMHLW group were significantly higher than those in the IOM group.

Table 5 shows the characteristics of the overweight women with the optimal weight gain recommended in the JSSO and IOM guidelines. There were no significant differences in these valuables between the two groups. Table 6 shows the perinatal outcomes of the overweight women with the optimal weight gain recommended in the JSSO and IOM guidelines (JSSO and IOM groups). The incidences of preterm delivery and low-birth-weight infant in the JSSO group were significantly higher than those in the IOM group.

Table 7 shows the characteristics of the obese women with the optimal weight gain recommended in the JSSO and IOM guidelines. There were no significant differences in these valuables between the two groups. Table 8 shows the perinatal outcomes of the obese women with the optimal weight gain recommended in the JSSO and IOM guidelines. There were no significant differences in these valuables between the two groups.

\section{Discussion}

Recently, there have been many underweight women who abhor a large weight gain during pregnancy in Japan associated with their slimming desire $[4,6]$. In particular, young Japanese women's strong desire to be thin has been pointed out as the underlying cause. In conjunction with these trends, the average birth weight in Japanese neonates has been becom-

Table 3. Clinical Characteristics of the Normal-Weight Women With the Optimal Weight Gain Recommended in the JMHLW and IOM Guidelines

\begin{tabular}{|c|c|c|}
\hline \multirow{2}{*}{ Characteristics } & \multicolumn{2}{|c|}{ Weight gain during pregnancy } \\
\hline & 7 - 12 kg (JMHLW guideline) & 11.3 - $15.9 \mathrm{~kg}$ (IOM guideline) \\
\hline Number & 2,136 & 1,348 \\
\hline \multicolumn{3}{|l|}{ Maternal age } \\
\hline$<20$ years old & $34(1.6 \%)$ & $29(2.2 \%)$ \\
\hline$\geq 40$ years old & $269(12.6 \%)^{*}$ & $112(8.3 \%)$ \\
\hline Primiparous women & $1,064(49.8 \%)$ & $695(51.6 \%)$ \\
\hline Maternal height $(\mathrm{cm})$ & $158 \pm 5.4$ & $159 \pm 5.3$ \\
\hline Maternal weight at pre-pregnancy (kg) & $51.7 \pm 5.3$ & $52.5 \pm 5.4$ \\
\hline Body mass index at pre-pregnancy & $20.6 \pm 1.7$ & $20.7 \pm 1.7$ \\
\hline Maternal weight at delivery (kg) & $61.3 \pm 5.5^{*}$ & $65.6 \pm 5.6$ \\
\hline
\end{tabular}

Data are presented as mean \pm standard deviation or number (percentages). ${ }^{*} \mathrm{P}<0.05$ vs. IOM group. JMHLW: Japanese Ministry of Health, Labour and Welfare; IOM: Institute of Medicine. 
Table 4. Perinatal Outcomes of the Normal-Weight Women With the Optimal Weight Gain Recommended in the JMHLW and IOM Guideline

\begin{tabular}{|c|c|c|c|c|c|}
\hline \multirow[b]{2}{*}{ Characteristics } & \multicolumn{2}{|c|}{ Weight gain during pregnancy } & \multirow[b]{2}{*}{ P-value* } & \multirow[b]{2}{*}{ Odds ratio } & \multirow[b]{2}{*}{$\begin{array}{l}95 \% \text { confidence } \\
\text { interval }\end{array}$} \\
\hline & $\begin{array}{l}7 \text { - } 12 \mathrm{~kg} \\
\text { (JMHLW guideline) }\end{array}$ & $\begin{array}{l}11.3-15.9 \mathrm{~kg} \\
\text { (IOM guideline) }\end{array}$ & & & \\
\hline Number & 2,136 & 1,348 & & & \\
\hline Hypertensive disorders & $143(6.7 \%)$ & $118(8.8 \%)$ & 0.03 & 0.75 & $0.58-0.94$ \\
\hline Gestational diabetes mellitus & $37(1.7 \%)$ & $19(1.4 \%)$ & 0.49 & 1.23 & $0.71-2.14$ \\
\hline Preterm delivery & $135(6.3 \%)$ & $51(3.8 \%)$ & $<0.01$ & 1.71 & $1.23-2.38$ \\
\hline \multicolumn{6}{|l|}{ Neonatal birth weight } \\
\hline$<2,500 \mathrm{~g}$ & $227(10.6 \%)$ & $87(6.5 \%)$ & $<0.01$ & 1.72 & $1.33-2.23$ \\
\hline$\geq 4,000 \mathrm{~g}$ & $7(0.3 \%)$ & $21(1.6 \%)$ & $<0.01$ & 0.21 & $0.09-0.48$ \\
\hline Postpartum hemorrhage $\geq 1,000 \mathrm{~mL}$ & $152(7.1 \%)$ & $155(11.5 \%)$ & $<0.01$ & 0.59 & $0.47-0.75$ \\
\hline
\end{tabular}

Data are presented as number (percentages). *P < 0.05 vs. IOM group. JMHLW: Japanese Ministry of Health, Labour and Welfare; IOM: Institute of Medicine.

ing lighter every year. Twenty to thirty years ago, the average birth weight in Japan was about 3,300 g; however, it has now decreased to be about $3,000 \mathrm{~g}$ and the proportion of low-birthweight infants in Japan has been increased to about 10\% $[4,6]$. Recently, in addition, some birth cohort studies in Japan have also been believed to be suitable for epidemiological studies to demonstrate the "Developmental Origins of Health and Disease (DOHaD)" indicating the developmental plasticity and the mismatch concept [6-8]. Based on these backgrounds, the importance of nutrition and weight gain during pre-pregnancy and pregnancy has now been widely recognized in Japan [6]. Therefore, examinations concerning the optimal weight gain during pregnancy in Japanese women are very important.

In the current study, we examined which guidelines indicate the optimal range of weight gain during pregnancy in pregnant Japanese women based on their BMI during pre-pregnan- cy. Although some bias seemed to be presented in the current study, we could not find whether or not the JMHLW guideline is more appropriate for the underweight and normal-weight pregnant Japanese women compared with the IOM guideline. Otherwise, the median range of the two recommended weight gains by the JMHLW and IOM guidelines may be suitable for the underweight and normal-weight Japanese women during pregnancy. In this study, because we could not assess weekly weight gain, it is unknown whether or not the less weight gain in the JMHLW group is the cause of the increased incidence of premature delivery and/or low-birth-weight infants. In the JMHLW group, the premature delivery associated with the increased low-birth-weight infants might increase before maternal weight gaining by other reasons. We understand that this may be one of some serious limitations in this study. In order to assess the optimal range of weight gain for underweight and/or

Table 5. Clinical Characteristics of the Overweight Women With the Optimal Weight Gain Recommended in the JASSO and IOM Guidelines

\begin{tabular}{lll}
\hline \multirow{2}{*}{ Characteristics } & \multicolumn{2}{c}{ Weight gain during pregnancy } \\
\cline { 2 - 3 } & $\leq 7 \mathbf{~ k g}$ (JSSO guideline) & $\mathbf{6 . 8}-\mathbf{1 1 . 3} \mathbf{~ k g}$ (IOM guideline) \\
\hline Number & 141 & 161 \\
Maternal height $(\mathrm{cm})$ & $158 \pm 6.0$ & $158 \pm 5.6$ \\
Maternal weight at pre-pregnancy $(\mathrm{kg})$ & $67.7 \pm 6.6$ & $67.1 \pm 6.4$ \\
Body mass index at pre-pregnancy & $27.2 \pm 1.5$ & $26.8 \pm 1.3$ \\
Maternal weight at delivery $(\mathrm{kg})$ & $71.3 \pm 6.7^{*}$ & $76.3 \pm 6.6$ \\
Maternal age & & \\
$\quad<20$ years old & $2(1.4 \%)$ & $1(0.6 \%)$ \\
$\quad 240$ years old & $21(14.9 \%)$ & $21(13.0 \%)$ \\
Primiparous women & $59(41.8 \%)$ & $61(37.9 \%)$ \\
Maternal height $(\mathrm{cm})$ & $158 \pm 6.0$ & $158 \pm 5.6$ \\
\hline
\end{tabular}

Data are presented as mean \pm standard deviation or number (percentages). ${ }^{*} \mathrm{P}<0.05$ vs. IOM group. JASSO: Japan Society for the Study of Obesity; IOM: Institute of Medicine. 
Table 6. Perinatal Outcomes of the Overweight Women With the Optimal Weight Gain Recommended in the JASSO and IOM Guidelines

\begin{tabular}{|c|c|c|c|c|c|}
\hline \multirow[b]{2}{*}{ Characteristics } & \multicolumn{2}{|c|}{ Weight gain during pregnancy } & \multirow[b]{2}{*}{ P-value* } & \multirow[b]{2}{*}{ Odds ratio } & \multirow[b]{2}{*}{$95 \%$ confidence interva } \\
\hline & $\begin{array}{l}\leq 7 \mathrm{~kg} \\
\text { (JSSO guideline) }\end{array}$ & $\begin{array}{l}6.8-11.3 \mathrm{~kg} \\
\text { (IOM guideline) }\end{array}$ & & & \\
\hline Number & 141 & 161 & & & \\
\hline Primiparous women & $59(41.8 \%)$ & $61(37.9 \%)$ & & & \\
\hline Hypertensive disorders & $25(17.7 \%)$ & $13(8.1 \%)$ & 0.01 & 2.45 & $1.21-4.95$ \\
\hline Gestational diabetes mellitus & $15(10.6 \%)$ & $9(5.6 \%)$ & 0.14 & 2.01 & $0.87-4.65$ \\
\hline Cesarean delivery & $45(31.9 \%)$ & $63(39.1 \%)$ & 0.23 & 0.73 & $0.45-1.17$ \\
\hline \multicolumn{6}{|l|}{ Neonatal birth weight } \\
\hline$<2,500 \mathrm{~g}$ & $15(10.6 \%)$ & $7(4.3 \%)$ & 0.04 & 2.62 & $1.06-6.44$ \\
\hline$\geq 4,000 \mathrm{~g}$ & $4(2.8 \%)$ & $2(1.2 \%)$ & 0.42 & 2.32 & $0.49-10.99$ \\
\hline Postpartum hemorrhage $\geq 1,000 \mathrm{~mL}$ & $17(5.0 \%)$ & $16(3.7 \%)$ & 0.58 & 1.24 & $0.61-2.53$ \\
\hline
\end{tabular}

Data are presented as number (percentages). ${ }^{*} \mathrm{P}<0.05$ vs. IOM group. JASSO: Japan Society for the Study of Obesity; IOM: Institute of Medicine.

Table 7. Clinical Characteristics of the Obese Women With the Optimal Weight Gain Recommended in the JASSO and IOM Guidelines

\begin{tabular}{|c|c|c|}
\hline \multirow{2}{*}{ Characteristics } & \multicolumn{2}{|c|}{ Weight gain during pregnancy } \\
\hline & $\leq 5 \mathrm{~kg}$ (JSSO guideline $)$ & 5.0 - $9.1 \mathrm{~kg}$ (IOM guideline) \\
\hline Number & 61 & 41 \\
\hline \multicolumn{3}{|l|}{ Maternal age } \\
\hline$<20$ years old & $1(1.6 \%)$ & $1(2.4 \%)$ \\
\hline$\geq 40$ years old & $8(13.1 \%)$ & $7(17.1 \%)$ \\
\hline Primiparous women & $25(41.0 \%)$ & $21(51.2 \%)$ \\
\hline Maternal height $(\mathrm{cm})$ & $158 \pm 6.6$ & $156 \pm 4.4$ \\
\hline Maternal weight at pre-pregnancy $(\mathrm{kg})$ & $83.5 \pm 9.5$ & $81.0 \pm 7.9$ \\
\hline Body mass index at pre-pregnancy & $33.5 \pm 3.0$ & $33.3 \pm 2.5$ \\
\hline Maternal weight at delivery (kg) & $84.3 \pm 7.8^{*}$ & $88.0 \pm 7.4$ \\
\hline
\end{tabular}

Data are presented as mean \pm standard deviation or number (percentages). ${ }^{*} \mathrm{P}<0.05 \mathrm{vs}$. IOM group. JASSO: Japan Society for the Study of Obesity; IOM: Institute of Medicine.

Table 8. Perinatal Outcomes of the Obese Women With the Optimal Weight Gain Recommended in the JASSO and IOM Guidelines

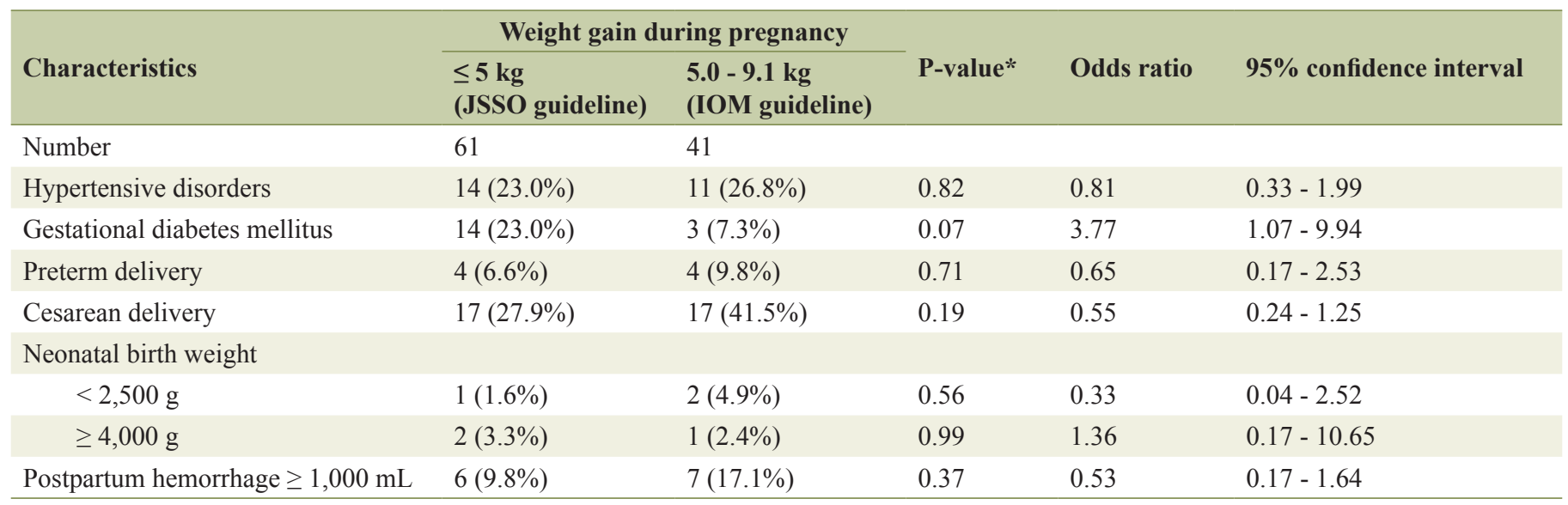

Data are presented as number (percentages). ${ }^{*} \mathrm{P}<0.05$ vs. IOM group. JASSO: Japan Society for the Study of Obesity; IOM: Institute of Medicine. 
normal-weight women during pregnancy, a larger prospective study will be required.

On the other hand, the IOM guideline seemed to be more appropriate for the overweight pregnant Japanese women compared with the JSSO guideline. In this study, the neonatal outcomes in these women seemed to be improved according to the optimal weight gain in the IOM guideline. To date, in Japan severe limit of weight gain has been seemed to be instructed for obese women for preventing dystocia [4, 9]; however, the optimal weight gain recommended by the IOM guideline seemed to be more suitable for the overweight Japanese women at least during pregnancy.

In this study, in addition, we could not well examine about the appropriate weight gain for obese pregnant Japanese women. One of some possible reasons leading to this observation may be the small sample size in the current study. Otherwise, in the obese women, the pre-pregnancy obesity itself might affect the perinatal outcomes more than the range of weight gain during pregnancy as previously reported $[1,2]$. Therefore, a larger prospective study will be also required to assess the optimal range of weight gain for obese women during pregnancy.

\section{Conclusion}

We should be more tolerant for the weight gain during pregnancy in Japanese woman than ever, especially in overweight women. A larger study may be needed.

\section{Conflicts of Interest}

The authors report no conflicts of interest. The authors are responsible for the content and writing of the paper. This study was supported by the Japan Health, Labour and Welfare scientific research subsidies: "Study on the support of nutritional management of pregnant women, mothers and infants".

\section{References}

1. Rasmussen KM, Yaktine AL, Eds. Weight gain during pregnancy: reexamining the guidelines. Washington, DC: National Academies Press; 2009.

2. Enomoto K, Aoki S, Toma R, Fujiwara K, Sakamaki K, Hirahara F. Pregnancy Outcomes Based on Pre-Pregnancy Body Mass Index in Japanese Women. PLoS One. 2016;11(6):e0157081.

3. Minakami H, Maeda T, Fujii T, Hamada H, Iitsuka Y, Itakura $\mathrm{A}$, Itoh $\mathrm{H}$, et al. Guidelines for obstetrical practice in Japan: Japan Society of Obstetrics and Gynecology (JSOG) and Japan Association of Obstetricians and Gynecologists (JAOG) 2014 edition. J Obstet Gynaecol Res. 2014;40(6):1469-1499.

4. Promotion Council for Healthy Parents and Children 21 (second edition) (in Japanese). Ministry of Health, Labour and Welfare, 2015. http://rhino3.med.yamanashi. ac.jp/sukoyaka2/english.html (June 15, 2016).

5. Japan Society for the Study of Obesity, Eds. Obesity in pregnant women (in Japanese). Japan Society for the Study of Obesity (JSSO) guideline 2016. 2016; p. 90-91.

6. Sata F. [Developmental Origins of Health and Disease (DOHaD) and Epidemiology]. Nihon Eiseigaku Zasshi. 2016;71(1):41-46.

7. Gluckman PD, Hanson MA, Beedle AS. Early life events and their consequences for later disease: a life history and evolutionary perspective. Am J Hum Biol. 2007;19(1):119.

8. Gillman MW, Barker D, Bier D, Cagampang F, Challis J, Fall C, Godfrey K, et al. Meeting report on the 3rd International Congress on Developmental Origins of Health and Disease (DOHaD). Pediatr Res. 2007;61(5 Pt 1):625629.

9. Suzuki S, Shin S, Araki T. Weight control during pregnancy (in Japanese). Obstet Gynecol Pract (Tokyo). 1996;45:927-932. 